\title{
Globe
}

Revue internationale d'études québécoises

\section{Le roman pour la jeunesse au Québec. Sa place dans le champ littéraire}

\section{Claire Le Brun}

Volume 1, numéro 2, 1998

URI : https://id.erudit.org/iderudit/1000512ar

DOI : https://doi.org/10.7202/1000512ar

Aller au sommaire du numéro

\section{Éditeur(s)}

Globe, Revue internationale d'études québécoises

ISSN

1481-5869 (imprimé)

1923-8231 (numérique)

Découvrir la revue

Citer cet article

Le Brun, C. (1998). Le roman pour la jeunesse au Québec. Sa place dans le champ littéraire. Globe, 1(2), 45-62. https://doi.org/10.7202/1000512ar

\section{Résumé de l'article}

Après un rapide état des lieux au niveau international, qui met en perspective le cas du Québec, l'article évalue la position du roman pour la jeunesse dans le champ littéraire québécois en observant successivement l'évolution de l'édition jeunesse; les instances de légitimation : couverture médiatique, prix, recherche universitaire; le point de vue des auteurs: leur image d'eux-mêmes et leur conception de l'écriture. En terminant, il s'interroge sur la signification d'un phénomène caractéristique du roman pour la jeunesse des décennies 80-90 : les références de plus en plus systématiques à la littérature générale. L'approche s'inspire globalement des travaux de Pierre Bourdieu, et plus particulièrement des Règles de l'art (1992). 


\title{
Le roman pour la jeunesse au Québec. Sa place dans le champ littéraire
}

\author{
Claire Le Brun \\ Université Concordia
}

La place de la littérature de jeunesse dans le champ littéraire est mal assurée : voilà une affirmation qui ne surprendra personne. Diversement reconnue selon les pays et les cultures, l'écriture destinée aux jeunes lecteurs consacre rarement un auteur. Le cas d'Astrid Lindgren en Suède demeure une exception". Le flou sémantique du syntagme «littérature de jeunesse» - désignant à la fois les lectures considérées a posteriori comme convenables pour la jeunesse et les ouvres conçues $a$ priori pour un public jeune - contribue à brouiller les perceptions et à décourager les tentatives de classification ${ }^{2}$. En outre, les

1 La créatrice de Pippi Långstrump (1945) - mieux connue des lecteurs francophones sous le nom de Fifi Brindacier - compte parmi les auteurs les célèbres, les plus lus et les plus appréciés en Suède, toutes catégories confondues. Ses œuvres ont été traduites en plus de soixante langues. Voir Ulla Lundqvist, Arbundrabets barn. Fenonemet Pippi Lingstrum och dess forruttsättningar [L'enfant de ce siècle : Le phénomène Fifi Brindacier, son origine et son influence], Stockholm, Rabén \& Sjögren, 1979 (avec un résumé en anglais : "The Child of the Centuryn); Mary Ørvig [éd.], En bok om Astrid Lindgren, Stockholm, Rabén \& Sjögren, 1977 (avec un résumé en anglais «A Book about Astrid Lindgrenu).

2 Sur cette question, voir la pertinente analyse de Danielle Thaler: «Littérature de jeunesse : un concept problématique», Canadian Children's Literature/Littérature canadienne pour la jeunesse, vol. 22, no 3, pp. 26-38.

Claire Le Brun, «Le roman pour la jeunesse au Québec. Sa place dans le champ littéraire», Globe. Revue internationale d'études québécoises, vol. 1, no 2, 1998. 
rapports qu'entretient la littérature de jeunesse avec les champs pédagogique et artistique ${ }^{3}$ tendent à remettre en question son appartenance au champ littéraire. Aussi nous concentreronsnous dans le cadre de cet article sur une seule catégorie, celle dont les rapports avec le champ littéraire paraissent les moins problématiques : le roman.

Pour commencer, un rapide état des lieux, au niveau international, permettra de mettre en perspective le cas du Québec. Puis, nous tenterons de cerner la place du roman pour la jeunesse dans le champ littéraire québécois en observant successivement l'évolution de l'édition jeunesse au cours des deux demières décennies; les instances de légitimation - prix, couverture médiatique, recherche universitaire -; le point de vue des auteurs : leur image d'eux-mêmes et leur conception de l'écriture. Nous terminerons par quelques commentaires sur un phénomène caractéristique du roman pour la jeunesse des décennies $80-90$ : les références de plus en plus systématiques à la littérature canonique, qui nous paraissent révélatrices de la quête de légitimité des auteurs. Notre approche s'inspire globalement des travaux de Pierre Bourdieu, et plus particulièrement des Règles de l'art.

\section{Perception de la littérature de la jeunesse chez les auteurs et chez les universitaires}

Dans un essai sur la poétique de la littérature de jeunesse paru en 1986, la chercheure israélienne Zohar Shavit consacre un chapitre à la mauvaise image qu'ont d'eux-mêmes les écrivains

3 Notamment dans le cas de l'album illustré, souvent considéré comme objet d'art et pièce de collection pour les adultes.

4 Pierre Bourdieu, Les Règles de l'art. Genèse et structure du champ Littéraire, Paris, Seuil, 1992. 
pour la jeunesse. ${ }^{5}$ Beaucoup d'entre eux souffrent du peu de reconnaissance de leur travail. Certains le considèrent comme une étape vers l'écriture pour les adultes; d'autres se voient imposer cette étape par leur éditeur. D'autres encore ont été définitivement, et contre leur gré, classés «auteurs pour la jeunesse». Shavit rappelle également que les romans pour la jeunesse paraissaient souvent sans nom d'auteur au XIXe siècle et que, tout au long du XXe siècle, le pseudonyme a été pratique courante.

L'auteur français Christian Grenier analyse le cas de quelquesuns de ses confrères ${ }^{6}$. Selon Grenier, la plupart d'entre eux n'ont pas choisi leur public : "Ils écrivent par besoin, par passion, comme d'autres nagent, chantent ou dansents. La rencontre de leur écriture avec les enfants ou les adolescents «n'est pas le résultat d'une démarche volontaire, mais le fruit d'un faux hasard.» ${ }^{7}$ Il cite le cas d'un auteur surpris de son succès auprès des jeunes lecteurs et ignorant résolument ces demiers. Un autre, qui affirme qu'il s'adresse aux adultes, s'avoue humilié d'être lu par des enfants. Pour un troisième, écrire à l'intention des jeunes est devenu une gageure depuis que son éditeur lui a promis qu'après deux romans publiés dans une collection jeunesse, il serait capable d'accéder à la littérature pour adultes. Quant à Grenier lui-même, son éditrice lui a révélé qu'il écrivait, sans le savoir, des romans pour les garçons de quatorze ou quinze ans. Le credo de Michel Toumier, pour qui le livre pour enfants représente l'état le plus achevé de l'écriture, n'est donc pas universellement partagé. Les témoignages rassemblés par Shavit et par Grenier mettent en lumière le rôle décisif des instances

5 Zohar Shavit, Poetics of Children's Literature, Athens-London, The University of Georgia, 1986.

6 Christian Grenier, «Les couleurs d'un siècle. Un carrefour d'enfances» in Denise Escarpit et Bernadette Poulou [éd.], Le Récit d'enfance. Paris, Éditions du Sorbier, 1993, pp. 159-168.

7 Op. cit., p. 160. 
éditoriales dans la naissance des «vocations» des auteurs pour la jeunesse. L'auteur pour la jeunesse semble plus souvent honteux que glorieux.

Voyons maintenant dans quels champs (pédagogique, littéraire, paralittéraire) se situe la recherche universitaire. Quatre tendances récentes donneront une idée des diverses conceptions. Le Britannique Peter Hunt est en faveur de recherches et de méthodes spécifiques à la littérature de jeunesse, d'une Childist critics, préconisant ainsi une fermeture du champ sur lui-même ${ }^{8}$. Zohar Shavit, mentionnée plus haut, qui applique la théorie du polysystème à la littérature de jeunesse, estime que le statut de cette littérature est analogue à celui de la littérature non canonique pour adultes, avec sa classification par sujets, par lectorat, par sexe? Quant au statut de ses auteurs, étant donné les liens de la littérature de jeunesse avec le système éducatif, Shavit le juge proche de celui du travailleur social ${ }^{10}$. Maria Nikolajeva, universitaire suédoise d'origine russe, disciple de Yuri Lotman, a une approche sémiotique ${ }^{11}$. Considérant que la littérature de jeunesse internationale est de plus en plus élaborée, elle démontre, par des exemples empruntés à différents pays, que le roman pour la jeunesse tend à se rapprocher du roman moderne ou post-moderne, et à rejoindre ainsi la littérature générale. Cette opinion est partagée par la Belge Ganna Ottavaere-van Praag, historienne de la littérature de jeunesse européenne, qui étudie les techniques narratives du roman pour la jeunesse contemporain ${ }^{12}$. Ces deux études, parues la même

8 Voir Peter Hunt, Criticism, Theory, and Children's Literature, London, Blackwell, 1991.

9 Op. cit., p. 33.

10 Op. cit., p. 35.

11 Maria Nikolajeva, Children's Literature Comes to Age, New YorkLondon, Garland Publishing, 1996.

12 Ganna Ottevaere-van Praag, Le Roman pour la jeunesse : approches, définitions, techniques narratives (1996), Bern, Berlin, Frankfurt/M., 
année, sont un appel sans équivoque à faire entrer la littérature de jeunesse dans la critique et la recherche littéraires générales. II apparait donc qu'il n'y a pas actuellement d'unanimité sur l'objet d'étude ni sur les méthodes. Coexistent deux tendances opposées : la revendication de la périphérie et les tentatives de rapprochement du centre du champ littéraire.

\section{La littérature de jeunesse au Québec}

Après cette mise en perspective, nous observerons le cas du Québec en commençant par un portrait de l'édition jeunesse. Après une relative stagnation dans les années 70 , ce secteur de l'édition québécoise a connu un développement sans précédent au milieu des années $80^{13}$. En 1979, la nouvelle revue Lurelu, vouée à la promotion de la littérature de jeunesse québécoise, titrait : «La commercialisation du livre de jeunesse québécois :

New York, Paris, Wien, 1996. Il peut être aussi intéressant de consulter : La Littérature pour la jeunesse en Europe occidentale (17501925). Histoire sociale et courants d'idées. Bern, Peter Lang, 1987, de la même auteure.

13 Il ne peut être question dans ce cadre restreint de faire un survol historique de la littérature de jeunesse québécoise au XXe siècle. Nous renvoyons à l'ouvrage fondateur de Louise Lemieux, Pleins feux sur la littérature de jeunesse au Canada frangais, Montréal, Leméac, 1972 et à la synthèse récente d'Édith Madore, La Littérature pour la jeunesse au Québec, Montréal, Boréal, 1994 (notamment la première partie : «L'Histoire de la littérature québécoise pour la jeunesse», pp. 15-54. Au cours des années 90, plusieurs mémoires et thèses ont été consacrés à l'histoire de l'édition québécoise pour la jeunesse. On suit également avec intérêt les recherches de Suzanne Pouliot et de Noëlle Sorin sur «L'Édition de livres pour la jeunesse» dans le cadre du Groupe de recherche sur l'édition du livre au Québec (GRÉLQ). 
une lueur au bout du tunnel ${ }^{14}$. Dix ans plus tard, le même périodique conclut : "Travailler dans le secteur jeunesse n'est plus, ou presque plus, marginal et des maisons bien établies ajoutent ce secteur à leurs activités éditoriales" ${ }^{15}$. En 1993, il va plus loin : "Voilà dix ans, seuls les audacieux se lançaient en littérature de jeunesse; aujourd'hui c'est un marché viable, très viable, presque un impératif. Pourquoi? Les libraires vous le diront : les jeunes Québécois lisent des romans québécois, contrairement à leurs aînés.» ${ }^{16}$ En effet, en 1991, les bibliothèques de Montréal ont enregistré plus d'un million de prêts de livres pour jeunes ${ }^{17}$. Si cette statistique n'indique ni la proportion d'œuvres québécoises ni le nombre total de prêts, elle n'en reste pas moins éloquente.

Cette constitution d'un bassin de lecteurs doit beaucoup aux efforts conjugués des enseignants, des concepteurs de programmes scolaires, des bibliothécaires et des organismes de promotion tels que Communication-Jeunesse ${ }^{18}$. Il faut également souligner le rôle très actif du fondateur des éditions La Courte Échelle, Bertrand Gauthier, qui a été le premier à mettre sur pied un système de diffusion efficace. La Courte Échelle se distingue par un souci constant de mise en marché sur la scène locale et à l'étranger. Ses livres sont traduits en une

14 Renée Rowan, «La commercialisation du livre de jeunesse : une lueur au bout du tunnely, Lurelu, vol. 2, no 1 (1979), pp. 3-6.

15 Michelle Provost et Lucie Julien, «Les années 80 . En partance pour les années 90 ), Lurelu, vol. 12, no 2 (1989), p. 9.

16 Suzanne Thibault, "Survol des collections de romans jeunesse", Lurelu, vol. 16, no 1 (1993), p. 4.

17 Suzanne Thibault, «Les Collections de romans pour la jeunesse et l'âge de leurs lecteurs", Lurelu, vol. 15, no 2 (1992), p. 36.

18 L'organisme Communication-Jeunesse a été fondé en 1971 (présidente-fondatrice : Paule Daveluy). Il a tenu son premier congrès en 1972 : «Création culturelle pour la jeunesse et identité québécoise». 
douzaine de langues et plus de la moitié des titres sont traduits ${ }^{19}$.

Les années 80 ont confirmé la présence des éditeurs pour la jeunesse au sein des industries culturelles. Les éditeurs publient de plus en plus de titres chaque année. En 1991, on a recensé 518 romans et recueils de contes ou de poèmes pour la jeunesse $^{20}$. En 1992, pas moins de 41 collections de romans visent les lecteurs débutants, intermédiaires et expérimentés ${ }^{21}$. Selon Lurelu, au milieu des années 90 , on pouvait avancer une moyenne de 10000 exemplaires vendus par titre, alors que, dans le même marché, un titre pour adultes se vendait bien à 2000 exemplaires $^{22}$. Même si l'on a pu observer un léger fléchissement à la fin de la décennie $90^{23}$, la littérature pour la jeunesse (surtout le roman, qui a éclipsé l'album au milieu des années 80 ) est l'un des secteurs de l'édition les plus rentables. De nouvelles collections ou même des maisons d'édition ${ }^{24}$ continuent à apparaitre. Il importe de signaler que le gros de la production est constitué par des auvres originales. Un seul éditeur accorde une large part de ses publications aux traductions de l'américain ${ }^{25}$.

19 Voir l'article d'Édith Madore, «Le Livre québécois pour jeunes sur le marché internationab), Lurelu, vol. 16, no 1 (1993), pp. 46-47. 20 Voir le bilan effectué en 1993 par Suzanne Thibault, op. cit., p. 4. 21 Voir l'enquête faite en 1992 par Suzanne Thibault, op. cit, pp. 3637.

22 Voir l'article de Michelle Provost et Suzanne Julien, op. cit, p. 9.

23 La chaîne de librairies Renaud-Bray - qui avait ouvert deux magasins exclusivement consactés aux livres et aux produits culturels pour enfants et adolescents - a considérablement réduit l'espace réservé à la littérature de jeunesse. Certaines maisons d'édition ont peu de visibilité dans l'ensemble des librairies.

24 Notamment celle de Robert Soulières, ancien directeur littéraire aux Éditions Pierre Tisseyre.

25 Il s'agit des Éditions Héritage. Selon Marie-Christine Thiffault, les traductions ne représentent que $10 \%$ du marché du livre pour la 
Pour donner une idée des instances de légitimation en littérature de jeunesse québécoise, nous examinerons successivement la couverture médiatique réservée au roman pour la jeunesse; les prix littéraires; et la place réservée à la littérature de jeunesse dans les programmes universitaires.

Au cours des années 80 , les chroniques littéraires des principaux quotidiens et de certains mensuels se sont ouvertes aux productions destinées à un jeune public. La télévision et la radio invitent occasionnellement un écrivain pour jeunes. Les praticiens du livre pour la jeunesse jugent cependant cette couverture insuffisante. Robert Soulières, auteur, directeur de collection, ancien directeur de la revue Lurelu, estime que ales médias sont assez silencieux à l'égard de la littérature de jeunesse. Sauf à Noël où elle sort du placard culturel $)^{26}$. Denis Côté, auteur prolifique et reconnu dans son sous-champ ${ }^{27}$, intitule un article d'humeur : "Vous écrivez pour les jeunes? Comme c'est cute! Et quand donc écrirez-vous un vrai livre?». Il y déplore la désinvolture des critiques : «Dans de nombreux médias, on semble croire que parler occasionnellement des livres pour les jeunes, c'est déjà leur faire honneur [...] Lorsqu'on traite de la littérature-jeunesse, point nécessaire d'être bien informé et

jeunesse au Québec, alors qu'en France la proportion est de $50 \%$ ("Le champ éditorial de la littérature de jeunesse au Québeo), communication présentée au colloque «Le champ littéraire de la jeunesse au carrefour de la recherche universitaire", Association canadienne-française pour l'avancement des sciences, Québec, 13 mai 1998).

26 Voir l'entrevue accordée par l'auteur à Yolande Lavigueur, «Robert Soulières ou l'Art de réaliser ses rêves», Lurelu, vol. 12, no 1 (1989), p. 18.

27 Nous désignons par «sous-champ» la littérature pour la jeunesse au Québec, à la fois sous-champ de la littérature québécoise et souschamp de la littérature pour la jeunesse internationale. 
encore moins d'être exhaustif.»" ${ }^{28}$ Bertrand Gauthier, écrivain et éditeur, s'en plaint également : "Comme écrivain pour enfants, je n'ai jamais droit à la critique, comme les autres créateurs. Si un critique lit mon demier livre, il dit qu'il se met dans la peau des enfants, ce qui est faux et partial.»)

Ces citations sont extraites de la revue Lurelu, la seule revue consacrée à la littérature québécoise pour la jeunesse, comme le proclame son sous-titre. Cette revue, qui a été fondée en 1978 sous les auspices de Communication-Jeunesse, assume une fonction d'auto-légitimation en présentant des entrevues d'auteurs, des recensions, des dossiers, des tribunes et des articles sur l'édition.

Les prix de littérature jeunesse sont relativement nombreux, compte tenu de l'exiguiité du sous-champ. Le plus convoité est celui du Gouverneur général du Canada, ouvert depuis 1987 à la littérature de jeunesse. Plus que les autres, il représente la légitimation, comme le montre ce commentaire des lauréats de 1988, David Schinkel et Yves Beauchesne : «La littérature de jeunesse se situe enfin au même rang que la littérature pour les adultes. Le livre de jeunesse est enfin reconnu comme appartenant à la littérature. Ce n'est plus un hors-d'œuvre, c'est une écriture en soi.» ${ }^{30}$

En 1993, puis en 1995, Suzanne Pouliot a dressé, de la situation de la littérature de jeunesse à l'université, deux bilans

28 Denis Côté, «Vous écrivez pour les jeunes? Comme c'est cute! Et quand donc écrirez-vous un vrai livre?», Lurelu, vol. 11, no 1 (1988), pp. 36-37.

29 Entrevue accordée à Marie-Jeanne Robin, «Rencontre. Bertrand Gauthien, Lurelu, vol. 4, no 4 (1981), p. 18.

30 Voir Monique Poulin, «David Schinkel et Yves Beauchesne. Le défi de l'écriture", Lurelu, vol 11, no 1 (1988), p. 22. 
qui n'ont rien perdu de leur actualite ${ }^{31}$. Elle constate que l'enseignement de la littérature de jeunesse dans les universités québécoises se fait essentiellement dans le cadre des programmes de bibliothéconomie et d'éducation. Bien que des maîtrises et des doctorats portent ou aient porté sur la littérature de jeunesse, les programmes consacrés à ce sous-champ de la littérature de jeunesse dans le cadre d'un département de littérature ont du mal à s'implanter ${ }^{32}$. Pouliot craint, à juste titre, que cette restriction de l'enseignement aux facultés d'éducation, aux écoles de bibliothéconomie et des sciences de l'information, ne limite les axes de recherche aux biobibliographies et aux applications didactiques. Elle note également que la littérature de jeunesse n'a même pas, dans la hiérarchie des littératures enseignées, un statut équivalent à celui des littératures dites «de l'exiguités" . En l'état actuel de la recherche universitaire sur la littérature de jeunesse québécoise, Daniel Chouinard distingue quant à lui trois axes : les ouvrages de référence (bibliographies, manuels, panoramas), la "dimension sociologique» et la

31 Suzanne Pouliot, «L'Université, lieu de légitimation pour la littérature de jeunessen, Lurelu, vol. 16, no 1 (1993), Pp. 53-56; "L'Enseignement universitaire et la littérature de jeunesse», Éclats, no 8 (février 1995), pp. 4-6.

32 Le Certificat de premier cycle en littérature de jeunesse, créé en septembre 1985 au Département d'études littéraires de l'Université du Québec à Montréal, a été fermé. L'Université du Québec à TroisRivières semble reprendre le flambeau à la fin de la décennie 90 , avec l'engagement de deux professeur/e/s spécialisé/e/s en littérature de jeunesse. L'histoire de l'enseignement de la littérature de jeunesse dans les universités québécoises est une suite d'avancées et de reculs. Pouliot conclut néanmoins son bilan de 1995 sur une note optimiste: «L'Institution littéraire et l'Institution universitaire avec leurs diverses instances de légitimation allouent de plus en plus de place à la littérature de jeunesse et à ses différentes manifestations, du moins depuis bientôt quinze ans.» (p. 6).

33 François Paré, Les Littératures de l'exiguité, Hearst, Le Nordir, 1992. 
"dimension littéraire»". Le codirecteur de la revue Canadian's Children Literature/Littérature canadienne pour la jeunesse $(C C L / L C J)$ voit poindre un changement de perception à l'égard de la littérature de jeunesse et une possibilité pour cette dernière de sortir du ghetto.

Il importe de signaler qu'il n'existe pas de revue québécoise universitaire spécialisée en littérature de jeunesse. Dans la revue canadienne bilingue $C C L / L C J$, fondée en 1975, les livres et les auteurs du Québec sont sérieusement recensés et étudiés; toutefois, le travail de cette revue est relativement peu pris en compte par les intervenants du sous-champ de la littérature de jeunesse au Québec.

\section{Le point de vue des auteurs pour la jeunesse}

Le point de vue des auteurs, leur image d'eux-mêmes et de leur travail, pourraient se résumer par une formule : grande mission et mal aimés. Les thèmes récurrents dans les entrevues sont les difficultés spécifiques de l'écriture pour la jeunesse, la somme de travail nécessaire, les plus grandes exigences du public-cible. Les déclarations des auteurs visent souvent à réfuter l'idée reçue selon laquelle l'écriture pour la jeunesse serait plus facile que la «vraie» écriture.

Sur les difficultés spécifiques de l'écriture pour la jeunesse, Monique LaRue, auteure reconnue en littérature canonique, dit : «Écrire pour les enfants : un délicat dosage de pédagogie et de littérature. [...] L'écrivain pour enfants est constamment pris entre l'écorce et l'arbre. Et quand on pense que certains

34 Daniel Chouinard, "La littérature de jeunesse au Québec. Orientations et valeurs de la recherche universitaire", Québec français, no 103 (automne 1996), pp. 84-86. 
confondent "auteurs pour les petits" et "petits auteurs"»". Sa consœur, Marie-Andrée Wamant-Côté, met le doigt sur une contrainte particulière, l'interdiction de jouer de l'intertextualité : «Écrire pour les enfants, voilà déjà une difficulté malgré ce que l'on croit à tort; quand on écrit pour eux, on ne peut jamais faire de clin d'ceil cultureh, ni faire référence à quelque chose.»"

S'agissant du travail exigé par la rédaction d'un roman pour la jeunesse, Bertrand Gauthier estime que «l'écriture pour les enfants ou pour les adultes, c'est pareil : de la création qui prend forme.» ${ }^{37}$ L'auteur croit que les sujets tabous sont les mêmes dans les deux cas : «le sexe, les rôles, la liberté, la spontanéité». François Gravel, auteur de romans pour adultes et pour jeunes, juge que «les exigences particulières de l'écriture pour la jeunesse demandent une réécriture plus serrée que les romans pour adultes.) ${ }^{38}$

Les auteurs soulignent aussi les plus grandes exigences du destinataire : "Alors que le roman pour adultes a une vie publique plus grande, avoue François Gravel, c'est pourtant à la sortie de mes romans pour jeunes que je suis le plus nerveux. Ce qui me donne le trac, c'est que je n'ai jamais autant lu qu'entre 10 et $15 \mathrm{ans}^{39}$. De là à conclure à la supériorité du public jeune, il n'y a qu'un pas. Raymond Plante juge qu'il est plus intéressant d'écrire pour les jeunes parce «qu'ils sont plus ouverts à la vie,

35 Monique LaRue, «Écrire pour les enfants. Un délicat mélange de pédagogie et de littérature», Lurelu, vol. 2, no 4 (1979), p. 6.

36 Voir l'entrevue accordée par l'auteure à Marie-jeanne Robin : «Entrevue. Marie-Andrée Warnant-Côté», Lurelu, vol. 6, no 3 (1984), p. 20. Les italiques sont de nous.

37 Voir l'entrevue accordée à Marie-Jeanne Robin, op. cit., p. 19.

38 Voir l'article d'Isabelle Crépeau, «Rencontre avec un drôle d'oiseau : François Graveb», Lurelu, vol. 15, no 1 (1992) , pp. 40-41. 39 Ibid. 
qu'on peut laisser plus de liberté à l'imaginations ${ }^{40}$. Robert Soulières ne souhaiterait pas écrire pour les adultes, sinon un recueil de poésie. Pour lui, l'écriture pour adultes n'est pas une "promotion» : les meilleurs écrivains devraient aussi être ceux qui s'adressent à de jeunes lecteurs ${ }^{41}$. On voit que le discours de défense des auteurs passe par la valorisation du destinataire. La hiérarchie des écrivains ne se fonde-t-elle pas sur la qualité sociale et culturelle du public touché $\hat{e}^{42}$ ?

Nous signalerons pour terminer une caractéristique de ce sous-champ de la littérature québécoise, qui se retrouve dans d'autres champs dominés ${ }^{43}$ le cumul de positions occupées par les agents : auteur-éditeur, éditeur-critique; auteure-directrice de collection; professeure-auteure, etc. En 1992, deux figures connues du sous-champ, Henriette Major et Dominique Demers, débattent de cette question dans les pages de Lurelu. La première affirme qu'«on ne peut pas être les deux à la fois». "Parce que nous sommes peu nombreux, explique-t-elle, nous devrions être d'autant plus vigilants" ${ }^{44}$. La seconde croit que "ceux qui aiment profondément les livres pour enfants finissent par s'engager dans plus d'un créneaus. Elle voit une source de richesse là où sa consœur perçoit un conflit d'intérêts ${ }^{45}$.

40 Entrevue accordée à Marie-Jeanne Robin, «Rencontre. Raymond Plante", Lurelu, vol. 6, no 1 (1983), p. 20.

41 Op. cit., p. 18.

42 Pierre Bourdieu, op. cit., p. 304.

43 Le fandom de la science-fiction constitue un bon exemple.

44 Henriette Major, "Quelques réflexions sur... les auteurs-critiques», Lurelu, vol. 15, no 1 (1992), p. 34.

45 Dominique Demers, «Ne tirez pas trop vite sur les critiques», Lurelu, vol. 15, no 2 (1992), p. 50. 


\section{Faire entrer la littérature dans le roman pour la jeunesse pour intégrer ce dernier dans le champ littéraire}

Depuis le toumant des années 90 , le roman pour la jeunesse, surtout le roman pour adolescents, multiplie les références à la littérature générale : mention d'auteurs et de titres, citations, résumés d'intrigues, parallèles avec des héros romanesques, parodies, pastiches, romans-hommages à un auteur, etc. ${ }^{46}$ L'une des meilleures réussites du genre est sans doute L'Étoile a pleuré rouge de Raymond Plante ${ }^{47}$ où la célèbre strophe de Rimbaud informe les réseaux métaphoriques d'un roman sur la violence des adolescents ${ }^{48}$. Les auteurs conviés au roman jeunesse appartiennent d'abord à la littérature française - roman et, de façon plus surprenante, poésie, Prévert et Rimbaud en tête de liste -, puis à la littérature québécoise ${ }^{49}$. Puis, hors francophonie, à des auteurs d'expression anglaise $e^{50}$. Interrogés sur ces procédés intertextuels, les auteurs les justifient par une volonté de mener

46 Voir notre article : «Edgar Alain Campeau et les autres. Le lecteur fictif dans la littérature québécoise pour la jeunesse (1986-1991)", $V$ oix et Images, no 55 (1993), pp. 151-165.

47 Raymond Plante, L'Étoile a pleuré rouge, Montréal, Boréal, Boréal Inter, 1994, no 28.

48 «L'étoile a pleuré rose au coeur de tes oreilles, L'infini roulé blanc de ta nuque à tes reins; $\mathrm{La}$ mer a perlé rousse à tes mammes vermeilles Et l'Homme saigné noir à ton flanc souverainy cité en exergue. $\grave{A}$ la page de remerciements, l'auteur indique : «Les citations en italique sont des extraits de poèmes d'Arthur Rimbaud.»

49 Par exemple : Jacques Godbout, Jacques Poulin, Anne Hébert, Félix-Antoine Savard, Paul Chamberland, Anne Hébert, Gabrielle Roy, Louis Fréchette, Claude Péloquin, Yves Thériault.

50 Par exemple : Charles Dickens, Lewis Carroll, Agatha Christie, Edgar Poe, J. D. Salinger, John Irving, Stephen King. 
leur public vers d'autres lectures ${ }^{51}$. Sans mettre en doute leurs intentions, nous voyons également, dans ces utilisations à différents degrés de la "grande littérature», des signes envoyés vers le centre du champ littéraire. Partageant la connaissance et l'amour des œuvres légitimées, les auteurs pour la jeunesse réclament une meilleure position. Parmi eux se trouvent d'ailleurs d'anciens auteurs pour adultes, qui ont trouvé un lectorat plus vaste chez les jeunes, mais qui souhaitent conserver ou retrouver leur légitimité d'antan.

L'usage de l'exergue, qui s'est généralisé dans les années 90 , nous parait être le signe le plus évident de cette quête de légitimation $^{52}$. Plus qu'au public-cible, qui ne le remarquera probablement pas, cet élément péritextuel n'est-il pas destiné à la communauté des lettrés ${ }^{53}$ ? L'exergue, «décoration ostentatoire sur la poitrine de l'auteun selon Antoine Compagnon ${ }^{54}$, nous parait être le label de littérarité que certains auteurs posent sur une production culturelle au statut contesté.

51 Voir l'article de Bruno Lemieux, «Littérature pour adolescents et intertextualité", Canadian Children's Literature/Littérature canadienne pour la jeunesse, vol. 22, no 1 (1996), pp. 31-37.

52 Voir notre article : «L'exergue comme procédé de légitimation du roman québécois pour la jeunesse (1982-1994)", Canadian Children's Literature/Littérature canadienne pour la jeunesse, vol. 20, no 3 (1994), pp. 14-26.

53 Selon Gérard Genette, «'épigraphe est à elle seule un signal, qui se veut indice de culture, un mot de passe d'intellectualitén (Seuils, Paris, Seuil, 1987, p. 149).

54 Antoine Compagnon, La Seconde main, ou Le Travail de la citation, Paris, Seuil, 1979, p. 337. 


\section{Bilan et prospective}

Au Québec, le livre pour la jeunesse est devenu liun des secteurs les plus lucratifs de l'édition. On ne peut manquer d'être frappé, dans les librairies, par la visibilité des productions locales. A l'intérieur des nombreuses collections, les textes originaux constituent la majorité, en regard des traductions et des rééditions. On trouve peu de textes destinés à l'origine à un public général et re-publiés dans des collections jeunesse.

La littérature québécoise pour la jeunesse est la littérature québécoise la plus lue, si l'on en croit les chiffres de vente et de prêt. En conséquence, plusieurs auteurs jouissent d'un certain capital économique. On dit même que quelques-uns peuvent vivre de leur plume, situation rare au Québec ${ }^{55}$. Mais le capital symbolique n'est pas obtenu pour autant . "Qui oserait dire, demande Denis Côté dans l'article d'humeur cité plus haut, que Ginette Anfousse et Raymond Plante ne sont pas de vrais écrivains? ${ }^{56}$ Personne, sans doute. Mais sont-ils nombreux, parmi les littéraires légitimes, à les considérer vraiment comme leurs pairs? Comme le rappelle Pierre Bourdieu, un des enjeux centraux des rivalités littéraires est le monopole de la légitimité littéraire [...] c'est-à-dire, entre autres choses, le monopole de dire qui est autorisé à se dire écrivain ou même à dire qui est écrivain et qui a autorité pour dire qui est écrivain.. ${ }^{57}$ Les auteurs jeunesse les plus lus et les plus achetés suscitent l'envie de leurs collègues de la littérature dite générale. Certaines critiques donnent même à penser que le roman pour la jeunesse risque d'être déconsidéré dans son ensemble par le succès commercial et le rythme de publication parfois effréné. Déjà quelques collections, parmi les plus prospères, sont accusées de recettes,

55 Voir Suzanne Thibault (1993), op, cit., p. 5.

56 Op. cit., p. 37.

57 Op. cit., p. 311. 
de traitement sommaire, de sur-utilisation des auteurs «maison».

Quoi qu'il en soit, à côté des auteurs "pour jeunes» adoptant la posture du "travailleur social»" d'auteurs, et parfois d'éditeurs, soucieux de se rapprocher du centre du champ littéraire. La Courte Échelle, éditeur spécialisé en littérature jeunesse, a lancé en 1996 une collection pour jeunes et moins jeunes adultes, la collection «16-96", susceptible d'intéresser les critiques de la littérature générale ${ }^{59}$. Enfin, pour compléter ce tableau de la littérature de jeunesse, il importe de prendre un peu de recul pour observer le phénomène culturel sous un autre angle. En effet, si le roman pour la jeunesse n'est pas encore arrivé à se tailler une place dans le champ littéraire québécois, il est en voie d'occuper un espace significatif dans le champ de la littérature de jeunesse intemationale par le mouvement de traductions vers l'anglais, l'espagnol, l'italien, l'allemand, le japonais, en passant par le basque et le catalan. On sait que les romans de la Courte Échelle ont été massivement traduits pour le Canada anglais. On sait moins, par contre, que Robert Soulières est lu en espagnol, Michèle Marineau en suédois, Raymond Plante en néerlandais, Denis Côté en danois, pour ne citer que quelques exemples. Peut-être certains auteurs

58 Nous reprenons l'expression de Zohar Shavit («social workem), op. cit., p. 35). Comme exemple de cette attitude, on peut citer la collection «Faubourg Saint-Rock», créée en 1991 aux éditions Pierre Tisseyre, où quelques auteurs situent leurs récits dans un décor commun et s'échangent les personnages, en peignant un "quotidien qui colle à la réalité des garçons et des filles d'aujourd'huiv) (quatrième de couverture). Sur cette collection, voir notre article : «Montréal pluriel au Faubourg Saint-Rock : Une littérature didactique pour le Québec des années 90), Studies in Canadian Literature/Études en littérature canadienne, vol. 21, no 2 (1996), pp. 49-61.

59 Des auteurs «maison», tels que Chrystine Brouillet, Marie-Danielle Croteau ou Stanley Péan ont signé les premiers titres. 
REVUE INTERNATIONALE D'ÉTUDES QUÉBÉCOISES

pour la jeunesse, devenus des vecteurs de la culture québécoise à l'étranger, acquerront-ils ainsi une légitimité et pourront-ils accéder à une meilleure position dans le champ littéraire québécois. 